\title{
2 \\ Mental Health at Work: Misconceptions and Missed Opportunities
}

\section{Introduction}

In 1942, the Medical Research Council's Industrial Health Research Board initiated an investigation, led by Dr Russell Fraser and Dr Elizabeth Bunbury, into neurotic illness as a cause of absence from work. Prompted by concerns about industrial efficiency during wartime, the research focused on light and medium engineering industries from Birmingham and Greater London and attempted to gauge the 'true incidence' of the condition and 'its effects on production'. ${ }^{1}$ Their study of 3,000 workers found that 9.1 per cent of male workers and 13 per cent of female workers had suffered from what was described as 'definite' neurosis. ${ }^{2}$ The number of male cases uncovered in this study was significantly higher than those that were to emerge later in studies during the 1950s and 1960s from general practice, which broadly suggested a female to male ratio of 2:1. Once again, a familiar feature of this study was that greater numbers of men were diagnosed with what Fraser described as 'disabling psychosomatic symptoms' (3.5 per cent of men and 2.1 per cent of women). When the figures are taken together, it would appear that psychological and psychosomatic illness was a significant problem for men as well as women.

Fraser's research methodology was progressive for its time. Unlike other studies that used sickness certificates alone as the basis for investigation, Fraser's study of workers included two clinical examinations: physical and psychological. Workers' home life and environment were also examined by a social worker so that information about domestic arrangements and leisure activities could be included. Employment sickness records were also consulted. Although the author acknowledged that the wartime context of the study meant that the findings 
might not reflect those in peacetime, he concluded that neurotic illness was an important cause of industrial disability among the workers studied. ${ }^{3}$ However, the research undertaken into health and work during the decades following the Second World War was shaped by broader cultural, political and economic factors and focused primarily on unemployment, physical and chemical hazards and absenteeism, underestimating the prevalence and impact of mental illness. ${ }^{4}$ The remainder of this chapter explores the various agendas that underpinned these debates and also examines the broader construction of masculinity that endorsed a machismo culture at work, preventing open discussion about male mental illness.

\section{Developments in occupational health}

As is well known, legislation governing workplace health and safety evolved over the nineteenth and twentieth centuries, largely in response to concerns about the risks posed to workers by hazardous materials and dangerous practices. As Vicky Long has noted, from the mid-nineteenth century, developments in industrial and occupational health were implemented in response to public concerns, but also shaped by the broader political and economic context. ${ }^{5}$ The series of Factory Acts passed by the British parliament from 1819 initially sought to mitigate the poor conditions endured by women and children. By 1855 , a rudimentary industrial medical service was introduced by law and, in 1895, notification of important industrial diseases, such as lead, phosphorous and anthrax poisoning, was introduced. ${ }^{6}$ A cornerstone of the developing legislation was the 1833 Factory Act, which established a range of provisos limiting the working hours of young persons of less than eighteen years of age and the appointment of factory inspectors with power to enforce regulations. ${ }^{7}$ Legislation towards the end of the nineteenth century required that workers in dangerous trades be examined by certifying surgeons who notified cases of occupational disease. Following the introduction of the Workmen's Compensation Act of 1897, many employers voluntarily appointed physicians as a means of protecting themselves against compensation claims. ${ }^{8}$ At the turn of the twentieth century, the state had built a statutory medical service for factory workers, provided by approximately 1800 part-time certifying factory surgeons (later known as factory doctors). ${ }^{9}$ Their remit was threefold: to examine young persons under eighteen years of age for fitness for work; to undertake examinations of persons employed in dangerous trades; and to investigate cases of notifiable industrial 
disease. Throughout the first half of the new century, workplace legislation continued to develop, adding to regulations about specific trades to cover entire production processes. However, by the late 1960s, as industrial technology advanced, the shortcomings of such a prescriptive approach to factory health and safety were becoming clear. Following the recommendations of the Robens Report in 1972, the Health and Safety at Work Act, 1974, fundamentally changed the principles of workplace health and safety. Based on the concepts of self-regulation, goal-setting and voluntary codes of practice, this new legislation placed the responsibility for workplace welfare on employers and employees. Such a brief overview of occupational health in Britain should however not be read as an uncomplicated development of state intervention. As Long argues in her account of the rise and fall of the healthy factory, industrial health 'evolved from the contested negotiations between trade unions, employers, the medical profession and the state, as each sought to achieve their objectives through an array of strategies' ${ }^{10}$ Periodically, approaches to the health and safety of the workforce were also influenced by the requirements of, and responses to, the broader global context: the growth and decline of the British Empire and the two World Wars.

From the early twentieth century, concurrent to developments in occupational health were modifications to working practices as the principles of 'scientific management' were applied increasingly to production processes. At the turn of the century, influential figures such as Frederick Taylor, Henry Ford and Frank and Lillian Gillbreth introduced the new concepts of piece-rates, time and motion studies, and automation. Originating in the United States (US) but later introduced in Britain by British industrial psychologists, the underlying principles of scientific management were that the correct selection of employees and appropriate methods of work were central to maximising production and improving the welfare of workers. In 1921, Charles Myers co-founded the National Institute of Industrial Psychology with Henry Welch to promote scientific management and ostensibly improve standards for workers. Myers stated in his influential text, Industrial Psychology (1929), that the aim of the field was to 'discover the best possible human conditions in occupational work'. ${ }^{11}$ In Britain, automated operation processes were introduced increasingly to a range of manufacturing industries that required very large outputs. ${ }^{12}$ Although one of the principles of scientific management was to improve standards of work and welfare for workers, from the 1940s, the impact of scientific management on employees in fact became a source of concern 
and debate. ${ }^{13}$ Whereas, during the inter-war period, debates had been dominated by concerns about physical and mental 'fatigue', the decades following the Second World War saw a shift in approaches to industrial medicine towards a focus on the psychological pressures of new working practices. ${ }^{14}$

Sarah Hayes has shown recently that the debate about automated processes was exceptionally polarised. Some industrialists argued that the new methods would lead to reduced demands on physical health and result in a less hazardous environment, while others feared the 'dehumanisation' of the workplace. ${ }^{15}$ Indeed, in 1959, concerns prompted the World Health Organization (WHO) to convene a study group on the problems of automation. Seen within the context of wider social change and technological development, the group noted that it was difficult to separate the effects of automation from other influences, such as management style. ${ }^{16}$ The tone of the report was tentatively positive and the group cautioned against the propensity towards pessimistic appraisals that 'warn humanity against the industrial hell towards which it is inexorably moving'. ${ }^{17}$ They argued, for example, that any risk of automated work being rendered 'meaningless' would be offset by less monotony and repetition. The report called for 'less hysteria', and concluded that more emphasis should be placed on preparation, education and 'responsible' media coverage. ${ }^{18}$ However, commentators would later protest that modern manufacturing processes did indeed lead to workers experiencing monotony, isolation and a lack of control, causing physical and psychological ill effects - ill effects that were ultimately treated surgically or pharmacologically and compensated financially. ${ }^{19}$ Ultimately, the polarised and conflicting debates presented employers with the opportunity to ignore the health implications of automated processes, despite evidence that the new practices impacted on the physical and mental health of workers. ${ }^{20}$ As I have argued in Chapter 1, the technical and medical model of intervention in health that prevailed, not only underplayed the importance of the psychosocial environment at work, but resulted in missed opportunities more broadly when it came to detecting the prevalence and causes of psychological illness in male workers.

\section{Absenteeism and sickness absence in post-war Britain}

From the 1960s, the topic of absenteeism had generated considerable debate among industrialists and psychologists. The term was used to refer to short-term employee absence that occurred without suitable 
notification and without official sanction by medical personnel, and it differed from sickness-absence, which was a formally certified period of time off work due to illness. Absenteeism implied 'voluntary' absence for one or two days and was interpreted as a way in which employees might take time off to avoid pressure, or in retaliation against the employer as an expression of job dissatisfaction. Despite widespread concern about the effects of absenteeism on production and efficiency, by the early 1980s, no satisfactory explanatory framework for the phenomenon had been found. ${ }^{21}$ Part of the problem stemmed from a lack of accurate records and difficulties identifying which absences were in fact 'voluntary'. ${ }^{22}$ In attempting to explore the issue, most commentators focused upon the individual employee's motives for taking unsanctioned time off work and examined factors such as age, sex, rank, wage and length of service. Some concluded that some workers were simply 'absence prone', implying that taking time off work was a habit. However, Professor John Chadwick-Jones, formerly Director of the Occupational Psychology Research Unit at Cardiff University, was critical of explanations that focused upon the individual employee's motives for taking short-term leave. Instead, he formulated a 'theory of absenteeism' as a social phenomenon, 'as part of a social exchange between employees and management'. ${ }^{23}$

Certainly, a number of clear and distinct patterns emerged from the literature. ${ }^{24}$ For example, short-term absenteeism was negatively associated with age (older workers were less prone to absenteeism than younger employees). ${ }^{25}$ Workers in lower ranks were more likely to be absent than those at managerial level, the assumption being that senior managers were either more assiduous or that the flexibility inherent in senior roles allowed personnel to attend to personal matters in their own time more easily. ${ }^{26}$ The most striking pattern was the observation that women had consistently higher rates of absence than men. In a well-cited article published in 1962 in the International Labour Review, Viviane Isambert-Jamati explored factors that might contribute to high numbers of female absenteeism. The author noted that the problem was related to the responsibility for dependent children and that this was manifest in figures that suggested married women took more time off work than widows and spinsters. ${ }^{27}$ The highest rates of absence indeed occurred in women between the ages of twenty-five and twenty-nine 'reflecting the fact that there were young children to be tended'. ${ }^{28}$ Supporting this explanation, other researchers noted that a great deal of female absence occurred during the morning, ${ }^{29}$ the theory being that women were required to undertake household chores and childcare 
responsibilities at this time of day. Isambert-Jamati's research indeed suggested that some women felt that employment contradicted what they saw as their 'proper social function'. When asked about their feelings towards their job, those who felt 'that their proper place would be, and should always have been, at home, [were] far more numerous in the group of frequent absentees than in the group of regular workers' ${ }^{30}$ Female labour turnover was also consistently higher than male, again accounted for by personal factors that affected women's ability to work, such as childbirth, sickness in the family and care of children or relatives. ${ }^{31}$ This high labour turnover often coincided with absenteeism and was particularly high during winter months, where families fell sick more often, and during the summer school holiday period. ${ }^{32}$ The study of daily variations of absence also exposed interesting patterns whereby workers were more likely to be absent on a Monday than on other days of the week. This trend was not new and had prompted commentators much earlier to formulate expressions such as 'Blue Monday', 'Colliers' Monday', 'Drunken Tuesday' and, for the fortnightly paid, 'Lazy Wednesday'. ${ }^{33}$

Debates on absenteeism thus focused on broad trends from scanty data and upon what might be done by the employer to minimise loss of productivity. Hilde Berhend noted in 1959, that by themselves, patterns and trends did not tell the researcher much about the causes of absenteeism. ${ }^{34}$ Although there was broad consensus that high rates of absenteeism might be associated in some way with expressions of low job satisfaction, she cautioned that no clear-cut frontier existed between sickness and psychological malaise, pointing out that 'psychosomatic diseases and voluntary absences may both represent escapes from an unbearable situation'. ${ }^{35}$ In a review of literature in 1973, ChadwickJones noted that a very small number of authors had considered disorders of personality and neurotic and psychosomatic illnesses as causes of absenteeism. Fraser's study of neurosis in factory workers and Helen Flanders Dunbar's Psychosomatic Diagnosis (1943) were cited as notable examples; however, Chadwick-Jones expressed surprise that 'this field should be so neglected'. ${ }^{36}$ Undoubtedly, the focus of literature upon individual motivations and attendance behaviour resulted in missed opportunities to uncover and expose psychological and psychosomatic disorders in men. As this chapter will argue, symptoms of 'distress' in men commonly presented as ill-defined disorders that might prompt a short spell of time off work. As authors writing about alcoholism in industry had also observed, much Monday morning absence was caused by heavy drinking over the weekend - a connection seemingly lost 
to those undertaking research into short-term absence. The situation was duly exacerbated by the focus on female workers and the fact that employees were often untruthful about why they had been absent. ${ }^{37}$

By the late 1960s, absence due to medically certified sickness also presented considerable problems in industry, costing, in economic terms, around $£ 400$ million a year. ${ }^{38}$ As with absenteeism, patterns of sickness absence followed some broad, recognisable trends, once again suggesting that women experienced more sickness absence than men. In 1968, statistics from the Office of Health Economics suggested that men insured under the national insurance scheme experienced 479 spells of certified sickness absence for every 1,000 men at risk. For women, the rate was higher at 520 per $1,000 .{ }^{39}$ The authors noted that the number of episodes of absence had increased at a moderately steady rate between the mid-1950s and the late 1960s, but that the trend was towards more frequent, but shorter spells of absence. ${ }^{40}$ Again, concerns were raised that uncertified absences of less than four days most likely made up a significant total of absence among the working population; however, no national statistics for these absences were available. ${ }^{41}$ Younger male employees appeared to have more short spells of sickness than older workers, while older male workers had longer spells that were less frequent. These patterns were confirmed in a range of employment arenas. ${ }^{42}$ Shift workers tended to have fewer episodes of sickness, but were more likely to be off work long-term. ${ }^{43}$ A number of researchers focused on the types of unique stresses experienced by workers in specific jobs, concluding that, despite higher stakes and high pressure, job satisfaction was more common in managerial positions. ${ }^{44}$ Sickness rates were greater among miners and quarry workers, whereas agricultural workers appeared to take less time off work. ${ }^{45}$ Among rural communities, the consensus was that farmers rarely sought medical advice, often ignoring sickness and disease until crisis prompted emergency care. A number of GPs who were interviewed for this project remarked that agriculture was very tough and that farming families during the 1950s and 1960s lived in primitive conditions. Remembering emergency home visits, one GP recalled that homes were desperately cold and that life was very hard: 'I mean sometimes I couldn't take my jacket off it was so cold . . . but lovely people. And very non-complaining. And things you never see nowadays - people with locked hips from osteoarthritis shuffling along, men in their fifties. ${ }^{46}$ Another doctor who spent his life practising in rural Devon concurred, recalling that farmers 'rarely complained of minor disorders' and because they were 'working on their own account, they would put up with a lot of - considerable physical 
symptoms, before they complain' ${ }^{47}$ The tough circumstances faced by farmers were reflected in the fact that suicide was more common among them than in other occupations. Roger Lea, who practised in a rural community, felt that farmers communicated very poorly and that they 'were lonely' sort of people: 'And if they got depressed, they just worked and they carried on. ${ }^{\prime 48}$ Suicide, according to another GP who lived in a farming community, was more common among farmers because, in terms of access to licenced firearms, they 'had the means to do so' ${ }^{49}$ Indeed, more recent research has suggested that, historically, farmers, veterinarians, doctors and those serving in the police force have been more likely to take their own lives - all occupations where the access to firearms or toxic substances might facilitate suicide. ${ }^{50}$

Drawing on data from the General Household Survey (1972), the sociologist Peter Townsend noted that unskilled men were three times more likely to suffer from 'limiting, longstanding illness, disability or infirmity' than professional men. From the survey, he observed that, in comparison with professional men, unskilled workers lost an average of four-and-a-half times as many days from work in the year, demonstrating 'the disadvantage of the partly skilled and unskilled professional classes' ${ }^{51}$ As UK unemployment began to rise during the 1970s, commentators suggested that an inverse correlation relation existed between morbidity and socioeconomic status. M. Harvey Brenner, who published extensively on the links between mortality, morbidity and the economy, argued that 'economic instability and insecurity increase the likelihood of immoderate and unstable life habits, disruption of basic social networks and major life stresses - in other words, the relative lack of financial and employment security of lower socioeconomic groups is a major source of their higher mortality rates' ${ }^{52}$

Patterns of illness shifted throughout the period from the 1950s to the 1980s, in part reflecting improved diagnosis and treatment, preventive measures and changes in the incidence of diseases. ${ }^{53}$ Days of work lost through respiratory tuberculosis, for example, decreased significantly between the mid-1950s and the mid-1960s, as did the incidence of pleurisy, anaemia and skin diseases. ${ }^{54}$ Peptic ulcers (diagnosed more frequently in men), although still a significant problem throughout the 1950s and 1960s, were decreasing in number. ${ }^{55}$ Agar and Raffle's study of London transport workers, published in 1975, suggested that diseases of the stomach and duodenum were decreasing among older workers, but increasing in younger men. ${ }^{56}$ The most noticeable trend, discernible in all studies of sickness absence, was the large rise in coronary heart disease, psychiatric diagnoses, musculoskeletal disorders and gastric 
disorders (other than peptic ulcer). ${ }^{57}$ Although authors formulated somewhat different methods of categorising these conditions, it is clear from research undertaken throughout the period that they became an increasingly important factor in sickness absence. Testimonies from GPs who were practising during the 1960s and 1970s suggest that gastric disorders and backache featured as the most common psychosomatic conditions. One GP who was interviewed for this book noted perceptibly that, although heavy lifting was indeed a genuine cause for musculoskeletal conditions, 'you also did get the feeling that some of this back pain was a metaphor for having a heavy load somewhere in their lives'. ${ }^{58}$ Another recalled: 'The commonest way of presenting was of course the backache . . . so you either took that at face value, and gave them a week's rest or something - or probed a bit further to find out what was going on. ${ }^{59}$ The study, Off Sick, published by the Office of Health Economics in 1971, utilised data from the mid-1950s and noted a large increase in numbers of workers absent through 'sprains and strains', 'nervousness debility and headache' and 'psychoneuroses and psychoses' ${ }^{60}$ Most research suggested that women were more likely to experience psychoneurosis - usually by a significant margin. Logan and Brooke's survey of sickness, for example, examined the number of illnesses, days of incapacity and consultations for selected diagnoses and found from their sample of 4,000 interviewees that the mean monthly prevalence rates in 1950 for psychoneurotic disorders and personality disorders in all ages over 16 were 106 for men and 155 for women. ${ }^{61}$ However, reflecting studies undertaken in general practice, a defining feature of all the research undertaken from the 1950s was the large number of men appearing in statistics for gastric disorders. Comparisons between studies are difficult because these symptoms were variously described in studies as gastro-intestinal disturbances, indigestion and epigastric pain. Nonetheless, there was clear evidence that men consulted their doctors, and frequently took time off work as a result of gastric symptoms. ${ }^{62}$

Taylor's study of oil refinery workers published in 1968, which won the Occupational Health Prize of the British Medical Association in 1967, was notable for its attempt at clarifying some of the complexities related to sickness absence. ${ }^{63}$ The study (exclusively among men, due to its focus on refinery workers) examined patterns of sickness absence among workers who were divided into groups of never sick, frequently sick and long-term sick. Findings were matched to a control group. The methodology, in many ways similar to Fraser's earlier study on neurosis, included information not only collected from documents and records, 
but also from interviews, health examinations, investigations into family and personal past, social background and present home circumstances. The research suggested broadly that, although the men in the frequently sick group had nearly three times as many spells of sickness absence as their matched controls, there was very little difference in the types of illness experienced. ${ }^{64}$ There were, however, three conditions that were significantly more common among both the frequently sick and long-term sick groups than the control, or never sick groups: nervous breakdown, peptic ulcer and incapacitating back-pain. ${ }^{65}$ Taylor was perceptive in employing a range of investigative techniques, which allowed his researchers to draw more meaningful conclusions from the data. Unlike studies drawn from occupational sickness records alone, his approach allowed researchers to gather a large amount of medical, social and psychological information about each worker, which could be examined for associations with sickness absence. As a result, in comparison to other studies, a considerable amount of male neurosis was revealed. ${ }^{66}$ Factors such as having had an 'unhappy childhood' and experience of parental divorce emerged as significant in the groups of men who were frequently off sick, as did the loss of a parent by death before the age of 60 , which proved influential in 40 per cent of men who were frequently sick, in contrast to 20 per cent of the controls. ${ }^{67}$

The fact that Taylor's study revealed appreciable numbers of men who were explicitly defined as diagnosed with neurosis, is significant enough; however, when these cases are combined with the large numbers of musculoskeletal disorders, gastritis and dyspepsia - and an intriguing group of 'ill-defined' disorders - it would be reasonable to suggest that a number of these diagnoses were psychosomatic presentations of psychological disorder. When it came to neurosis, back-ache and peptic ulcer, Taylor had begun to make connections between a worker's social circumstances, his history, his medical diagnosis and absence patterns. However, his study lacked further analysis of gastric disorders other than ulcers, and made nothing whatsoever of conditions described as 'ill-defined'. This was somewhat surprising given that numbers in this category equalled those diagnosed with neurosis at 21.4 per cent of those frequently sick. A significant 30 per cent of frequently sick men appeared in the data for gastritis and dyspepsia, and for each of these conditions, cases in the control group were very small. ${ }^{68}$ As Chapter 1 suggests, there was certainly a consensus among GPs that gastric disorders were a common psychosomatic presentation among men. One doctor remembered a male patient who presented annually with peptic ulcer symptoms, although no organic cause was ever found: 
It later transpired that his symptoms mainly occurred in the early part of the year. And it was eventually decided that, because of his job, he had a lot of critical things to produce by April the 1st, and it was probably anxiety leading up to that that produced his symptoms. And he tried all sorts of things - antacids were about the only thing available then ... Then I think, after April the 1st, or from sort of May time onwards, it all abated. And all right for the next nine months. ${ }^{69}$

Authors interested in occupational health outside Britain were more forthcoming about drawing associations between repeated absence, neurosis and peptic ulcer. Some went further and drew a direct correlation between neurosis, heavy drinking and stomach disorders. One Australian study of repeated sickness absence in a range of occupations specifically noted: 'Neurosis, smoking and peptic ulcer, found to be linked with drinking, and the physical consequences of drinking to excess, no doubt contributed to the liability of the drinker to be absent repeatedly. ${ }^{170}$ The author of this research unequivocally stated that social factors, such as conjugal failure, drinking and other 'personal maladjustment', contributed to repeated sickness absence. ${ }^{71}$ The same author, in a study of neurosis among male telegraph workers in Australia, found that one-third (33 per cent) of the 516 workers who were examined were considered to have, or to have had, disabling neurosis. ${ }^{72}$ Most subjects mentioned more than one influence as being contributory to their symptoms. Among personal and domestic reasons, those most commonly cited were family ill health, money worries and marital discord. Occupational influences included inability to cope with the job, monotony and job dissatisfaction. ${ }^{73}$ Similar concerns were raised by contributors to an international, interdisciplinary series of symposia on society, stress and disease that took place through the 1970s. In the fourth of a resulting series of publications edited by Lennart Levi, Sweden's first Professor of Psychosocial Medicine, two Swedish authors examined stress and strain among Scandinavian, white-collar workers. ${ }^{74}$ They drew strong correlations between high levels of mental strain, the use of sedatives and tranquillisers, gastric disorders and nerves. The study did not include analysis of gendered patterns of illness, but nevertheless made the explicit observation that workers who reported psychological reactions to mental strain at work had 'a much higher frequency of medical complaints, above all in the form of gastric and nervous troubles' ${ }^{75}$ Their conclusions were that psychological and psychosomatic reactions were likely to occur simultaneously. ${ }^{76}$ 
All studies of mental illness in industry were hampered by the same methodological problems that affected epidemiological studies in general practice. As was demonstrated in Chapter 1, there was no clear definition of what 'mental illness' actually meant, and the classification of symptoms differed widely between studies. Writing later in 1980, a group of social psychologists from the University of Sheffield noted that there had been a complete lack of reliable empirical work on the subject because researchers had been unable to define or conceptualise adequately mental health or mental illness and that there had been a lack of valid measures for use with work populations. ${ }^{77}$ Terms such as 'nervous breakdown' and 'neurosis' were often used interchangeably, with no clear account of the symptoms included. ${ }^{78}$ Up until the late 1970s, in studies of occupational health, the term depression was rarely used, although some researchers referred to 'depressive neurosis', with no full explanation about what this inferred..$^{79}$ In many cases, depression and a whole host of other psychological symptoms were subsumed under the broad heading of neurosis. Some investigators included psychosomatic symptoms in their research, while others discarded them altogether. ${ }^{80}$ Those who excluded them invariably revealed fewer numbers of men since they were more likely to present with somatic symptoms. Other research included psychosomatic symptoms only if they presented concurrently with psychological symptoms that conformed to the WHO's definition of a mental 'case'. ${ }^{81}$ In 1957, making a case for the importance of epidemiological studies at work, R. S. F. Schilling argued that observing patterns of disease in groups might be the only way of detecting some occupational hazards and their influence on health..$^{82}$ However, he conceded that, particularly in relation to neurosis, there were numerous problems with the relatively simple association of cause and effect in much industrial disease, which had inevitably led to a narrow concept of industrial medicine. ${ }^{83}$ Schilling raised concerns about studies undertaken at work, where treatment records were likely to be 'an unreliable mix of minor accidents and illness'. Furthermore, he noted that whether or not a worker reported for treatment depended on many things: 'Some make light of minor ailments; others make the most of them. ${ }^{84}$ The personality of medical staff and the prospect of loss of wages were seen as important factors that might influence a worker's decision to seek treatment. Calling for simpler and surer methods of assessing psychological illness, Shilling noted that many errors occurred in observing clinical signs and taking histories of symptoms so that 'much that is recorded may be unreliable' ${ }^{85}$

Accurate sickness trends were also very difficult to trace through certificated medical absence since a great deal of controversy surrounded 
the question of medical certification, its value and effect. ${ }^{86}$ In order to claim sickness benefits from the Department of Health and Social Security, workers usually needed to be certified as unfit for work by a medical doctor, usually a GP. However, it was generally accepted that GPs' training did not equip them with the ability to measure an employee's capacity for work. ${ }^{87}$ As this chapter and the oral history testimonies throughout this book suggest, 'diagnoses suggestive of neurosis [were] often vague, and because in the eyes of some physicians and patients, a stigma attaches to mental disorder a more acceptable symptomatic diagnosis may be given on the certificate' ${ }^{88}$ One GP interviewed as part of this research recalled that it 'was a real . . real problem, having a psychiatric illness on your note. So . . . we would call a psychiatric illness a physical one, because that was acceptable, and it was acceptable to their friends and their boss and everything else' ${ }^{89}$ Likewise, a senior professor of general practice concurred: 'Because the man didn't want to be labelled as psychological, the doctor would go along with it. ${ }^{90}$ The same GP remembered that one of his patients, a senior executive, drove over thirty miles outside the local vicinity to collect his prescription, for fear that someone might discover he was being prescribed psychotropic drugs. ${ }^{91}$ The situation not only caused problems for patients, but also for GPs who were often placed in a difficult situation when deciding whether or not to issue a sickness certificate. One GP, contributing to a symposium on absence from work in 1969, noted that 'workmen do not usually want to bother a doctor' and that it was 'not part of the general practitioner's function to maintain industrial discipline or morale'. Indicating how unsatisfactory the whole process appeared to be, he added: 'There was a time in my career when I occasionally tried to refuse people certificates - but in the interest of my coronary arteries, I have given up arguing. ${ }^{92}$

Certainly, evidence suggests that workers were often reluctant to discuss the causes of short-term absence with their employers and that some men refused to take part in workplace health investigations. ${ }^{93}$ Those who did give consent to participate were often impervious to advice about health. An investigation during the 1960s, which explored the attitudes of senior staff in industry towards health investigations, noted that advice on smoking, drinking and eating habits was seldom received positively, possibly due to the employee's 'reluctance to remember advice which he found unpalatable'. ${ }^{94}$ Moreover, studies that featured groups of men who, in statistical terms, appeared to be rarely ill often exposed interesting ambiguities. The 'never-sick' group in Taylor's oil refinery study, for example, 'almost without exception . . . denied 
that there was anything wrong with their health, their home or their work' ${ }^{95}$ This prompted Taylor to question whether such responses were untruthful or whether these individuals simply lacked insight into their own health and social circumstances.

As Chapter 1 illustrated, concerns about the rise of nervous disorders and 'stress-related' illness emerged in part from broader anxieties about a rapidly changing world and the impact of conflict and cultural upheaval on mental and physical health. ${ }^{96}$ The eclectic range of physiological, psychological and psychosocial theories that emerged during the post-war period each claimed their own position within the debate about whether causes could be traced to the social environment or found within the individual. When it came to studies in industry, regardless of the perspective taken by investigators, productivity was primarily the motive for research. A study of long-term sickness among British civil servants undertaken during the late 1960s, for example, was borne from rising concerns about efficiency and occupational health. ${ }^{97}$ The survey was interpreted by the service's medical advisor, the epidemiologist Daniel Thomson, who showed 'little sympathy for employees and limited awareness of contemporary research on the potential impact of work on health'. ${ }^{98}$ Thomson was preoccupied with individual rather than corporate responsibility and described stress reactions in workers as 'largely of their own making' caused by personal shortcomings. ${ }^{99}$ Stress-related ill-health, according to Thomson, was not necessarily the product of greater pressure at work, but instead, the product of 'lowered stress thresholds', offering employees 'a convenient means of avoiding seemingly unbearable pressures' ${ }^{100}$ Absenteeism was seen as a 'broader malaise affecting modern society' ${ }^{101}$ Unsurprisingly, the study of civil servants exposed a familiarly gendered pattern of sickness absence: women were more likely to be off sick than men, and, while male workers were more likely to experience heart disease, women were more prone to mental illness. As Jackson has argued, although these patterns might well have reflected the difficulties experienced by women in balancing work and domestic responsibilities, they also betrayed common assumptions about gender that were woven into debates about industrial health and sickness. This is particularly interesting, since physiological studies of stress undertaken by the pioneering stress researcher, Hans Selye, uncovered very little difference between the sexes. Indeed, he maintained that stress reactions were universal and non-specific. ${ }^{102}$ It is clear from the civil servant study and from wider studies on occupational health, that a preoccupation with efficiency and the methodological problems associated with the collection and analysis of medical 
data, did much to hamper the potential to expose and explore male psychological and psychosomatic illness.

\section{Masculine culture in the workplace}

Although the framework of industrial and psychological investigations into workplace health was unhelpful in the sense that it often missed opportunities to uncover cases of male mental illness, additional factors exacerbated the problem. As Arthur McIvor has shown, during the postwar period 'the workplace was an important site for the incubation and forging of male identities' ${ }^{103}$ The 'essence' of masculinity, McIvor notes, with particular reference to the heavy industries, has been associated most often with physical prowess, toughness, homophobia, risk-taking and a lack of emotional display. ${ }^{104}$ Masculinity at work has been the subject of much historical debate in recent years and scholars rightly point out that neither masculinity nor femininity were fixed constructs; instead, 'a range of masculinities and femininities coexisted around the traditional breadwinner and housewife paradigm'. ${ }^{105}$ As Eileen Yeo has shown, masculinity is also 'fractured by class, race and ethnicity in settings where some versions of manhood are privileged and others subordinated' ${ }^{106}$ Nevertheless, in workplace culture the discourse of the 'hegemonic hard man' was the most influential. ${ }^{107}$ As McIvor has illustrated so strikingly with his oral histories of the heavy trades, working-class masculinities were nurtured in the tough street culture of the neighbourhood and in dangerous, dirty and physically exhausting work. ${ }^{108}$ This often 'brutal' world was mediated by the camaraderie of the workplace: black humour and repartee - and by heavy smoking and drinking outside work. ${ }^{109}$

Machismo behaviour had implications for both physical and mental health. McIvor suggests that there were two co-existing 'degenerative pressures upon health and workers' bodies': capitalist exploitation and masculine values. ${ }^{110}$ Risk-taking at work was an important feature of male working-class culture, and it is not easy to determine precisely why workers took risks with their health - whether it was in order to impress their peers, or because they felt pressured by management. ${ }^{111}$ David Walker has argued that machismo culture should not be seen as simply 'male strutting', but instead as 'emerging from and acted out as a consequence of the exploitation of the worker at the point of production'.112 In Walker's opinion, the overriding need to earn a sustained income meant that workers did not necessarily 'seek out' danger, but were made to accept hazardous working conditions. ${ }^{113}$ It is certainly the case, for example, that when safety equipment was first introduced in 
construction, steel works, mining and shipyards, many employees initially resisted wearing them. ${ }^{114}$ Nick Hayes has shown, with particular reference to the construction industry, that improvements in welfare provision for workers 'debased the accepted currencies of physical endurance and self-provision'. ${ }^{115}$ Unions noted that many operatives thought there was 'something cissy about safety and fanc[ied] themselves as tough guys'. ${ }^{116}$ Ultimately, for construction workers, life on site might have been 'harsh, uncertain and dangerous', but it was also 'informal, manly and self-defining'. ${ }^{117}$ Whatever complex motives lay behind risk-taking culture at work, it is clear that men were socialised into overcoming instinctual fears and apprehensions so that 'working in poor, dangerous conditions became the norm'. ${ }^{118}$

Central to workplace masculine culture was the importance of not appearing 'weak', which was manifest in oral testimonies and autobiographies of workers from the mid-twentieth century. Being 'at loggerheads' with management in the coal mining industry, for example, was a marker of the 'stoic struggle' against exploitative managers, employers and foremen. ${ }^{119}$ Within Scottish mining communities, older miners encouraged youths to avoid any display of emotion, and this was viewed as training to be 'hard men'. ${ }^{120}$ As Johnston and McIvor observed in their study of the Clydeside heavy industries: 'Any sign of weakness, emotion and vulnerability could lead to being pilloried: the butt of jokes, scathing banter, vicious nicknames and sometimes very public humiliation. ${ }^{121}$ Such attitudes were widely evident in the oral histories of doctors. Richard Stanton, a retired GP from Devon, recalled that he had to be very careful talking to men about psychosomatic symptoms "because the reaction would be "So you think I'm a hypochondriac?". . . it's all to do with the macho thing for men, isn't it'. ${ }^{122}$ Similarly, Sarah Hall, a GP with extensive medical experience in the East End of London, recalled that penetrating beneath the hard surface of market workers from Smithfield and Covent Garden was often very difficult:

They make ridiculous jokes, really stupid jokes. They know they're being stupid. It's their way of maintaining their mood. So they've got this amazing front on them that's really hard to get through. And they'd be like, in the consulting room: 'Hi love, how are you?' You know. And, so they'd be loud and cheerful. And it was very, very difficult to get past that . . . it was that 'matey' thing. ${ }^{123}$

Indeed, this machismo culture meant that many men resolutely refused to visit the doctor. GPs practising in the West Country observed that 
farmers were particularly reluctant to seek help. One doctor recalled that 'the farming fraternity, the hunting, shooting, fishing fraternity, were far more stoical and prepared to self-treat, and, when they came our way, one was usually more impressed by their symptoms'. ${ }^{124}$ Another noted specifically that farmers appeared to encounter problems with depression in their middle years, but that 'they sometimes never appeared with any symptoms - until they committed suicide'. ${ }^{125}$

During the 1950s and 1960s, a whole generation of men also brought the experiences of war to their working lives. Pat Ayres noted in her study of masculinities in post-war Liverpool: 'The manhood of those returning from serving abroad in the army of merchant service had been tested in the most overt way.' ${ }^{126}$ Post-war, the demands of national service ensured that large numbers of young men continued to experience the discipline of the army. Michael Roper, in his study of management, observed that military service had a significant influence on the men he interviewed: 'The physical hardships and discipline . . . had educated them in the cult of toughness . . . masculinity was won through . . . having learnt what discipline meant [and] was sustained by ritual purgings of the "feminine" parts of themselves.'127 Roper's study of managers also illustrates how the experiences of white-collar workers differed from 'hard men' working in heavy industry and manufacturing. Managers described 'a constant struggle to quell suspicions that they were unmanly or "soft"' and 'graded management hierarchies according to the level of aggression required to perform at each level'. Workers often felt they had 'failed to assert a sufficiently "hard" masculinity' ${ }^{128}$ The cult of toughness was manifest not only in the stories told by men, but also more literally 'embodied' in workers' postures, gestures and firm handshakes - and in their appearance, for example, with closecropped hair. ${ }^{129}$ On the shop floor, new Fordist practices, automation and conveyor-belt production were viewed as less 'manly' than traditional methods. However, as Ayers argues, the workplace remained an exclusively male milieu and, consequently, constructions of masculinity adapted and were remodelled 'in order to accommodate change without damaging men's sense of themselves as true men'. Ultimately, the experience of 'manliness persisted' ${ }^{130}$

\section{Occupational health}

Historical reflections on occupational health and industry in Britain since the Second World War have exposed the complex political agendas at play between employers, employees, the government and trade 
unions. Central to the argument made in this chapter is the suggestion that the ability to identify and observe male psychological illness at work was obscured by these agendas and compounded by a construction of masculinity characterised by toughness and a lack of emotional display. In dealing with the topic of health at work, there has been hot debate among historians about the role of trade unions in occupational health. Critical interpretations suggest that union policies have tended to neglect the health of the worker in favour of a focus on wages, job security and financial compensation policies. ${ }^{131}$ Johnston and McIvor argue, for example, with reference specifically to the risks of asbestos in Scotland, that the unions absorbed and reflected the macho attitudes of their dominant male workforce, rather than vigorously challenging this high-risk workplace health culture. ${ }^{\prime 32}$ However, alternative interpretations, including more recent analyses by Johnson and McIvor on workers' respiratory illness, suggest that the unions played a pivotal role in working to protect members. ${ }^{133}$ Joseph Melling, while not exonerating the unions, argues that they acted 'within constraints imposed upon them by other actors as well as their own members'. ${ }^{134}$ According to Melling, the trade unions were often working with limited information about health risks and were prompted to balance any risks against those associated with loss of earnings or reduction in employment that might accompany rigorous safety standards. ${ }^{135}$ However, when it came to psychological illness, the trade unions were reluctant to intervene. Vicky Long has shown that during the 1930s, healthcare workers increasingly viewed the Trades Union Congress (TUC) as an organisation legitimately interested in the provision of healthcare and that a number of psychologists were keen to collaborate on investigations into workers' mental health. J. R. Rees, for example, Medical Director of the Institute of Medical Psychology at the Tavistock Clinic, was keen for the TUC to fund work at the institute, arguing that psychoneuroses were an important focus area for research since they caused one-third of all sickness from industry. However, these appeals were met with indifference, suggesting that trade unions may have been reluctant to accept that their workers' health problems may have had a psychological basis. ${ }^{136}$ It is interesting that, despite a broad paradigm shift in discussion away from physical fatigue towards debates about the psychological health of workers, the TUC was reluctant to engage with debates about psychoneuroses, 'wary of the stigma still attached to mental illness'. ${ }^{137}$

During the immediate post-war period, opportunities to investigate the impact of mental illness among workers were also hampered by elemental questions about who should be responsible for occupational 
health in the first place. In her extensive exploration of political debates about industrial health, Long notes that the expansion of medical personnel in factories during the Second Wold War 'gave rise to the belief that a state industrial medical service would be inaugurated after the war in tandem with the new NHS'. ${ }^{138}$ However, despite the TUC's efforts to secure such a service, the demands for this provision were resisted. ${ }^{139}$ The Ministry of Health argued that 'industrial workers had no health needs that could not be met by the general health-care services', and suggested that industrial healthcare provision would simply duplicate that which would be available on the NHS. ${ }^{140}$ The situation was duly exacerbated by the shortage of doctors with training and experience in industrial health - a fact that ultimately buttressed the arguments put forward by the Ministry of Health. The Industrial Hygiene Service that was eventually established employed 'technical experts' to ensure the health of the working environment. In so doing, focus shifted firmly away from the importance of preventative measures. ${ }^{141}$ Thus, opportunities to investigate male health were almost certainly lost in the politics of occupational health. As McIvor notes, the state's over-reliance on scientific discourse, the lack of a preventative programme and the consequent narrow focus on specific occupational diseases did little to erode high-risk workplace health cultures. ${ }^{142}$

As the material in this chapter and the subsequent chapter on alcohol abuse suggests, debates about the health of workers in other countries often focused more openly on the problems of mental illness and alcoholism in industry. There are no straightforward explanations for this; however, it is clear that in the international arena, different cultural values and contrasting approaches to state intervention in matters of health resulted in alternative models of occupational health. Scandinavian researchers, for example, were prominent in studies undertaken on occupational health, and often cited in British journals. ${ }^{143}$ The 'Nordic model' of welfare has been the topic of widespread debate and is held by some as an egalitarian and equitable example of state-regulated healthcare, funded by taxation. ${ }^{144}$ Leaving the politics of this debate aside, if the origins of the Nordic welfare model are considered, it is possible to see why workers' health featured prominently in debates. As Mary Hilson has shown, the Scandinavian social democratic welfare state that developed was characterised by a number of guiding principles. Primarily, there was of course the expectation of state involvement via taxation. However, this was assisted by a strong work ethic where 'willingness to work' was a condition for receiving benefits. Social reform thus was seen to integrate the working classes and prevent 
social unrest, primarily through 'creating the means for individuals to support themselves'. ${ }^{145}$ Scandinavian welfare was closely connected to the pursuit of economic efficiency and intended to be prophylactic, in that it provided the state with the means to create the 'good' society. Bolstered by deep-rooted Nordic values of individual responsibility for social welfare through sobriety, education and respectability, the prophylactic policy also extended to the realms of healthcare. Under the influence of a strong temperance movement, almost all Nordic countries, for example, experimented with some form of state control of alcohol during the early twentieth century. ${ }^{146}$ This preventative approach was more likely to stimulate productive research into the health of workers and stood in marked contrast to the disease model that dominated in Britain. Hilson rightly warns against oversimplified histories that assume the same trajectory for all Nordic countries, and cautions against whig histories of the state welfare model. Nevertheless, the Nordic model has undoubtedly been characterised by collectivism and conformism, where welfare states have evolved peacefully without the open conflict that has been more typical in other countries such as the Britain and USA. Moreover, within the Nordic model, the trade unions have been more central to debates and held close links with the social democratic leadership.

On the international scene, state intervention in health was not necessarily the primary factor to influence the direction of debates about the psychological health of workers. For a set of completely different reasons, the concept of prevention also became embedded in psychiatric approaches in the USA. Here, psychiatric thought was shaped by the mental hygiene movement, which became influential from the first decade of the twentieth century. The National Committee for Mental Hygiene (NCMH) was founded in New York in 1909 by a number of leading psychiatrists and influenced in particular by Clifford Whittingham Beers (1876-1943) and Adolf Meyer (1866-1950). Beers, who had spent several years himself in psychiatric institutions following suicide attempts and mental breakdown, wrote a book entitled $A$ Mind that Found Itself, which was published in 1908. Beers intended this publication to be 'a prelude to the formation of a national movement', which initially sought to improve institutional care. However, following Meyer's involvement, the objectives shifted significantly to encompass a broader move towards the promotion of health and the prevention of mental illness. ${ }^{147}$ Surveys sponsored by the NCMH suggested that 'the bulk of individuals requiring psychiatric treatment were not in institutions' and the journal Mental Hygiene, which was first published in 1917, stressed 
the importance of preventative medicine, education and research into factors that affected the mental health of the population. ${ }^{148}$

Early interest in industrial psychology thus, in part, stemmed from the influence of the mental hygiene movement. ${ }^{149}$ The importance of social and psychological factors in mental health at work was certainly reflected in the work of leading researchers such as the psychologist Elton Mayo (1880-1949), who emphasised the key role of the environment and human interaction in his authoritative text, The Human Problems of an Industrial Civilization, published in 1934. Increasingly, research that appeared in the American Journal of Psychiatry stressed the need for industrial physicians to 'concern themselves with the recognition of emotional factors underlying behaviour, which so frequently resulted in inferior output, high sickness rates, high labor turnover and absenteeism'. ${ }^{150}$ Building on this approach, many large corporations developed psychiatric programmes for their workplaces, and feature articles in national newspapers attracted the attention of the general public. ${ }^{151}$

In addition to existing concerns about absenteeism and automation (that were commonplace in Britain), American literature regularly encompassed research on alcoholism, neurotic reactions and the more general application of psychiatry to business and industry. ${ }^{152}$ Many of these publications included case studies of interviews and medical examinations with male workers who were affected by emotional distress and psychosomatic illness. Writing in 1959, for example, W. Donald Ross, in his book Practical Psychiatry for Physicians, described an exchange with a coal worker complaining about shortness of breath. Having made passing reference to the death of a fellow miner in a rock fall, the worker 'skirted around' the subject to focus again on his symptoms. When it was suggested that the death of his friend might have bothered him a good deal, the worker admitted that he 'hadn't liked to talk about it' but had 'worried considerably about it' and was then able to consider the impact of this trauma on his own feelings and anxieties about the hazards of mining. ${ }^{153}$ This book included an entire chapter on 'psychophysiological problems' in which Ross explored a host of somatic presentations that might be caused by emotional distress, including: musculoskeletal disorders, asthma, chest pain, gastrointestinal disturbances, ulcerative colitis and spastic colon. ${ }^{154}$ Another chapter examined job stresses that were specific to workers of different grades, and the author observed that executive workers were not only prone to anxiety and depression, but also to a host of psychosomatic illnesses which included indigestion, headaches and hypertension. ${ }^{155}$ It is notable that Ross, despite what might seem like a progressive approach, still 
subscribed to the notion that women were biologically predisposed to emotionality, arguing that changes in attitude among women 'spring from the different motivations at different times in the ovarian cycle'. ${ }^{156}$ Nevertheless, as Chapter 3 of this book will demonstrate, such texts did not shy away from sensitive issues such as alcoholism among workers in marked contrast to formal discussion in Britain that was in almost complete denial that a problem existed at all.

A focus on preventative measures in the USA was almost certainly also connected to the system of employer-sponsored health insurance that developed from the 1930s. As David Blumenthal has noted, two historic events prepared the way for the emergence of employer-sponsored insurance. The first was President Franklin D. Roosevelt's decision not to pursue universal health coverage after his election in 1932, and the second was a series of federal rules enacted during the 1940s and 1950s on how employer-sponsored insurance should be treated with regard to federal taxes and in labour negotiations. ${ }^{157}$ Roosevelt's decision created an opportunity for commercial insurers to step in and sell insurance to employers to provide protection for workers faced with the growing cost of illness. Simultaneously, the federal government's decision to limit employers' freedom to raise wages, resulted in employers expanding benefits to workers as a package to attract employees. Finally, in 1954, contributions made by employers to the purchase of health insurance for employees were ruled as non-taxable income to workers. ${ }^{158}$ Consequently, private health insurance soon became an established feature of American life which appeared to diminish 'the need for government action but also had spawned a strong new insurance industry with a stake in the status quo'. ${ }^{159}$ When compared to a system of universal coverage, opinions about the benefits and drawbacks of an industrysponsored approach have been deeply polarised. However, leaving the politics aside once again, it is perhaps easy to see why, within a healthcare system that developed within the employment arena, employersponsored research and prevention programmes were more common in the USA. Contrastingly, in Britain, the NHS came to be seen as the most appropriate arena for the diagnosis and treatment of conditions that were not specifically related to health risks at work.

\section{Reflections}

Writing in the British Journal of Industrial Medicine in 1985, Rachel Jenkins, Professor of Epidemiology and Mental Health Policy, stated that: 
Industrial policy makers, scientists and the informed public have, until recently, concentrated their attention on three major workrelated areas: unemployment, physical and chemical health hazards and absenteeism. Mental illness in the workforce has been of subsidiary interest and attention has focused on the separate issues of whether work is an aetiological factor in mental illness. ${ }^{160}$

Jenkins' article outlined many of the methodological anomalies and cultural biases that had hampered research into the mental health of workers since the Second World War. She noted, for example, that previous studies had tended to rely on the diagnoses given by GPs on sickness certificates and suggested that the estimates derived from these investigations were notoriously low. In her own research on psychiatric morbidity among executive officers in the Civil Service, Jenkins' respondents filled out the General Health Questionnaire (GHQ) and participated in a psychiatric interview. Results were then combined with analysis of employment sickness records and followed up twelve months later. The GHQ was intended specifically as a screening tool to detect those who either already had a psychiatric disorder or were at risk of developing one. ${ }^{161}$ Early empirical studies using this tool suggested a good level of consistency and reliability, and investigators also sanctioned its use in employment settings. ${ }^{162}$ Using this methodology, Jenkins found that psychiatric symptoms were common among her respondents and that there was 'no pronounced difference between the sexes'. ${ }^{163}$ Jenkins underlined the fact that official statistics underestimated the extent of the problem, since they relied on the GPs ability to diagnose psychiatric illness. Citing work from Goldberg published in 1976, she argued that 'it is known that between a third and a half of psychiatric disorders presenting in general practitioners' surgeries remains undetected by the general practitioner.'164 Additionally, she maintained that, 'since stigma and discrimination may accrue to receipt of a psychiatric diagnosis, the general practitioner may avoid writing such a diagnosis on the certificate of an employed person'. ${ }^{165}$ The stigma associated with mental illness, she argued, prompted many individuals to present in primary care with a physical symptom since this was more socially acceptable than a psychiatric illness. Friends, relatives and GPs, she maintained, 'often share[d] this view'. ${ }^{166}$

By the 1980s, social scientists interested in occupational health were also drawing attention to the problems with previous studies. A group of psychologists at the Medical Research Council's Applied Psychology 
Unit in Sheffield, for example, argued that the problem with empirical work thus far had been the inability to define or conceptualise the terms 'mental health' and 'mental illness' and the fact that there had been a lack of demonstrably valid measures for use with work populations. ${ }^{167}$ Cary Cooper, who went on to become an internationally renowned professor of organisational psychology, argued that previous studies had been undertaken within single disciplines and that interdisciplinary work between psychology, sociology, medicine and management might expose more useful insights. ${ }^{168}$ In a study of occupational sources of stress, co-authored with colleague Judi Marshall, Cooper articulated increasingly widespread concern that the field had been constrained by methodological problems related to the measurement of stress and psychological illness. They remarked that too few studies had explored psychosomatic presentations of psychological illness and added that finding adequate control groups for research had been problematic. ${ }^{169}$ Drawing attention to the fact that a number of extra-organisational factors, such as family problems and financial difficulties, also contributed to stress and mental illness, Cooper and Marshall called for more research into the broader relationship between home and working life. In their 'model of stress at work', the authors concluded that there were multiple sources of stress at work, which, combined with the individual characteristics of the worker and personal pressures, could result in both physical and mental symptoms: hypertension, depressed mood and escapist drinking. ${ }^{170}$

In a continuation of her work on civil servants, Rachel Jenkins also published in 1985 a study of psychiatric morbidity and its association with labour turnover. Once again employing the GHQ and a system of interviews, the study incorporated not only investigation into aspects of job motivation and satisfaction, but also factors related to social support networks, relationships and financial circumstances of participants. Jenkins maintained that it was misguided to attempt to understand labour turnover by focusing primarily on workers' attitudes towards their employment. Instead, she concluded that minor psychiatric illness (including alcohol abuse) was an important cause of labour turnover for both men and women and that a range of social, psychological and economic factors should be included as possible causes. ${ }^{171}$ Calling for further research, her concerns reflected the sentiments of a growing number of researchers working in fields allied to medicine and psychiatry that were keen to further their understanding of such a complex problem. Lennart Levi, writing in 1981, indeed suggested that official data on stress at work was 'only part of the story' because there 
were 'other indicators of a bad person-environment fit at work and elsewhere, such as alcoholism, suicide, mental and psychosomatic disorders'. These, he argued, were very common phenomena, yet no reliable data existed concerning the components of the total situation at work and outside it. ${ }^{172}$ As numerous commentators were beginning to point out, interest in mental health was far greater in some other countries, in particular the USA, where industry had begun to develop a range of innovative programmes to investigate and manage psychiatric disorders among workers. However, in Britain, research remained 'scanty', and, as Jenkins noted, psychiatry as a whole appeared to show little interest in the field of mental health in the workplace. ${ }^{173}$

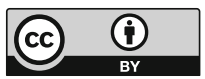

Except where otherwise noted, this work is licensed under a Creative Commons Attribution 3.0 Unported License. To view a copy of this license, visit http://creativecommons.org/licenses/by/3.0/ 